\title{
INTEGRAL OPERATORS OF HARMONIC ANALYSIS IN SPACES DEFINED IN TERMS OF LOCAL CHARACTERISTICS OF FUNCTIONS
}

\author{
Sadiq K. Abdullayev ${ }^{1}$, Elchin A. Mammadov ${ }^{2} \S$ \\ ${ }^{1}$ Institute of Mathematics and Mechanics of ANAS \\ B. Vahabzade str. 9, AZ 1141, Baku, AZERBAIJAN \\ ${ }^{1,2}$ Department of Mechanics - Mathematics \\ Baku State University \\ Acad. Zahid Khalilov str. 23, AZ 1148, Baku, AZERBAIJAN

\begin{abstract}
In the paper we consider boundedness of subadditive operators from a rather wide class containing in particular, singular integral operators, Riesz and Bessel potentials, maximum functions, fractional-maximum functions, Poisson integral, associated with LaplaceBessel differential operators, in new scales of Banach spaces, introduced in terms of integral characteristics like $\Omega_{p}$.
\end{abstract}

AMS Subject Classification: 42B10, 42B20, 42 B35

Key Words: subadditive operators, Riesz potential, generalized shift operators, LaplaceBessel differential operator

\section{Introduction}

Study of integral operators in terms of characteristics like $\Omega_{p}$ starts from the papers $[1,2,3]$ where the operators of Fourier classic harmonic analysis are considered.

\begin{tabular}{lcc}
\hline Received: & March 9, 2017 & (c) 2017 Academic Publications, Ltd. \\
Revised: & March 28, 2017 & url: www.acadpubl.eu \\
Published: & May 23, 2017 & \\
${ }^{\text {Correspondence }}$ author &
\end{tabular}


In these studies, establishment of estimates connecting these characteristics of the image with the same characteristics of the pre-image of the operator from the certain class is a starting point.

In the present paper similar researches are carried out for the operators of Fourier-Bessel's harmonic analysis.

Undoubtedly, singular integral operators (SIO), maximum function, Riesz and Bessel potentials, etc. are powerful working apparatus of Fourier-Bessel's harmonic analysis (associated with Fourier-Bessel transform).

Here the convolutional structures generated not by ordinary but by some special shift $T^{y}$ (the so-called generalized shift [4]) adapted to Fourier-Bessel transform in some coordinates of the point, are considered.

Note that in different issues of mathematics, for examples, when studying boundary values of $B$-harmonic functions associated with Laplace-Bessel's differential operator

$$
\begin{gathered}
\Delta_{B_{m+k, k}}(x)=\sum_{j=1}^{m} \frac{\partial^{2}}{\partial x_{j}^{2}}+\sum_{j=m+1}^{m+k}\left(\frac{\partial^{2}}{\partial x_{j}^{2}}+\frac{\gamma_{j}}{x_{j}} \frac{\partial}{\partial x_{j}}\right), \\
x \in R_{m+k, k}^{+} \quad \gamma_{m+1}>0, \ldots, \gamma_{m+k}>0
\end{gathered}
$$

there arises a necessity of studying potentials and SIO generated by the generalized shift $T_{\gamma_{n, k}}^{y}$ in one-dimensional case introduced by B.M. Levitan [4,5] and called the operator of generalized or Bessel shift (see the papers of I.A. Kipriyanov [6], I.A. Kipriyanov, M.I. Klyuchanchev [7]).

In I.A. Kipriyanov and L.A. Ivanov's paper [8] it is proved that the solution of the equation $\Delta_{B_{m+k, k}}(x)=f(x)$ is an integral operator like the cylindrical potential

$$
\begin{aligned}
u(x) \equiv I_{B}^{\alpha}(f)(x)= & \int_{R_{m+k, k}^{+}}|y|^{\alpha-n-\left|\gamma_{k, m+k}\right|} T_{\gamma_{n, k}}^{y}(f(x)) y^{\gamma_{k, m+k}} d y \\
& 0<\alpha<m+k+\left|\gamma_{k, m+k}\right|
\end{aligned}
$$

(when $\alpha=2$ ) called the generalized Riesz potential. The problem of obtaining a priori estimates, in principle is reduced to estimation of these generalized Riesz potentials and their appropriate derivatives (singular integrals). In this connection they have introduced the spaces $L_{p, \gamma_{m+k, k}}$.

Establishment of weight $L_{p, \gamma_{m+k, k}}$ estimates is one of the contensive directions of investigation of integral operators of Fourier-Bessel's harmonic analysis. 
For SIO generated by the generalized shift $T_{\gamma_{n, k}}^{y}$ (in the case $k=1$ ) this problem was first studied in the paper of I.A. Aliyev and A.D. Gadjiev [9], where only the case of radial weights was considered.

The case of non-radial weights was first considered in the papers of S.K. Abdullayev, N.R. Karamaliyev [10], S.K. Abdullayev, A.A. Akperov and M.K. Kerimov [11,12] when the weight function is dependent only on one coordinate of space variable. The case of certain class of general weights is considered in S.K. Abdullayev, E.A. Gadjiev and F.A. Isayev's paper [13].

In the present paper, in particular these results are taken to the case of weights dependent on arbitrary set of $s \in\{1, \ldots, m+k\}$ coordinates.

To this end, following [3], a class of operators $\bar{K}_{\gamma_{n, k}}(p, q)$ and integral characteristics $\Omega_{p, \mu_{n, k}}^{\left(s^{x}\right)}(u, \xi)$ of locally summable functions $u(x), \quad x \in R_{m+k, k}^{+}$are introduced and in their terms, the estimations connecting $\Omega_{p, \mu_{n, k}}^{\left(s^{x}\right)}$ characteristics of the image with $\Omega_{p, \mu_{n, k}}^{\left(s^{x}\right)}$ characteristics of the preimage of the operator from the class $\bar{K}_{\gamma_{n, k}}(p, q)$ are proved.

The obtained results become a starting point when studying these operators in different scales of Banach spaces determined in the terms of introduced characteristics, one of which is the scale of weight $L_{p, \gamma_{m+k, k}}$ spaces.

The classes like subadditive operators $\bar{K}_{\gamma_{n, k}}(p, q)$ containing in particular singular integral operators (SIO), maximum and fractional maximum functions, the Riesz and Bessel potentials and other operators majorized by convolution type integral operators with generalized shift $T_{\gamma_{n, k}}^{y}$, were introduced for studying a wider class of Harmonic analysis operators from unique position. But we note that owing to generality of the approach, the results obtained by us contain the case of ordinary shift in all coordinates, more exactly the case of Fourier harmonic analysis operators.

\section{Some Denotation and Preliminary Information}

Let $R^{l}$ be dimensional Euclidean space and $m, k \geq 0$ be integers,

$$
\begin{array}{r}
n=m+k \geq 1, \quad R_{m+k, k}^{+}=\left\{\left(x_{1}, \ldots, x_{m+k}\right) \in R^{m+k}: x_{m+i}>0, i=1, \ldots, k\right\} \\
R_{m+0,0}^{+} \equiv R^{m} . \\
-T_{\gamma_{n, k}}^{\gamma}(u(x))=c_{\nu} \int_{0}^{\pi} \ldots \int_{0}^{\pi} u\left(x^{\prime}-y^{\prime},\left(x_{m+1}, y_{m+1}\right)_{\alpha_{1}}, \ldots,\left(x_{m+k}, y_{m+k}\right)_{\alpha_{k}}\right)
\end{array}
$$




$$
\prod_{i+1}^{m+k} \sin ^{\gamma_{m+i}-1} \alpha_{i} d \alpha_{i}
$$

be a generalized shift operator (GSO) generated by the Laplace-Bessel operator

$$
\begin{gathered}
\Delta_{B_{m+k, k}}(x)=\sum_{j=1}^{m} \frac{\partial}{\partial x_{j}^{2}}+\sum_{j=m+1}^{m+k}\left(\frac{\partial^{2}}{\partial x_{j}^{2}}+\frac{\gamma_{j}}{x_{j}} \frac{\partial}{\partial x_{j}}\right), \\
x \in R_{m+k, k}^{+} \quad \gamma_{m+1}>0, \ldots, \gamma_{m+k}>0, x^{\prime}, y^{\prime} \in R^{m}, \\
x=\left(x, x_{m+1}, \ldots, x_{m+k}\right), \quad y=\left(y, y_{m+1}, \ldots, y_{m+k}\right) \\
\left(x_{m+i}, y_{m+i}\right)_{\alpha_{i}}=\sqrt{x_{m+i}^{2}-2 x_{m+i} y_{m+i} \cos \alpha_{i}+y_{m+i}^{2}}
\end{gathered}
$$

$i=1, \ldots, k, C_{\nu}$ be a normalizing multiplier.

Further, assume

$$
\begin{gathered}
\gamma_{n, k}=\left(0, \ldots, 0, \gamma_{m+1}, \ldots, \gamma_{m+k}\right) \in R_{m+k, k}^{+}, \quad\left|\gamma_{n, k}\right|=\sum_{i=1}^{k} \gamma_{m+i}, \\
y^{\gamma_{n, k}}=\prod_{i=1}^{m+k} y_{i}^{\gamma_{i}}=y_{m+1}^{\gamma_{m+1}} \ldots y_{m+k}^{m+k}, \quad d \mu_{n, k}(y)=y^{\gamma_{n, k}} d y, \quad \text { if } y \in R_{m+k, k}^{+} .
\end{gathered}
$$

In denotation, $\gamma_{n, k}, n$ indicates the dimension of this vector, while $k$ is the amount of its positive coordinates.

Remark 1. If $k=0$, we assume $\gamma_{n, k}=(0, \ldots, 0) \in R^{m}, T_{\gamma_{n, k}}^{y} f(x)=$ $f(y-x)$ is an ordinary shift, and $d \mu_{n, k}(y)=d y$.

When $n=m+k \geq 2$ and $s \in\{1, \ldots, m+k-1\}$ we partition the space $R_{m+k, k}^{+}$of the points $x=\left(x_{1}, \ldots, x_{m+k}\right)$ into the direct sum of the space $R_{s, k_{s}}^{+}$ of the points ${ }_{s} x=\left(x_{n_{1}}, \ldots, x_{n_{s}}\right)$ with coordinates $x_{n_{1}}, \ldots, x_{n_{s}}$ where

$$
\left(\left\{n_{1}, \ldots, n_{s}\right\} \cap\{m+1, \ldots, m+k\}\right), \quad 1 \leq n_{1}<\ldots<n_{s} \leq m+k
$$

and the space $R_{n-s,\left(k-k_{s}\right)}^{+}$of the points ${ }_{s} x^{\prime}$ so that $x=\uparrow\left({ }_{s} x,{ }_{s} x^{\prime}\right) \in R_{n, k}^{+}$(for denotations see [14]).

Let $m_{s}=\operatorname{rang}\left(\left\{n_{1}, \ldots, n_{s}\right\} \cap\{1, \ldots, m\}\right)$, then $m_{s}, k_{s}$ are integers such that $0 \leq m_{s} \leq m, 0 \leq k_{s} \leq k$ and $m_{s}+k_{s}=s$.

In these denotations we also assume

$$
m_{s}^{\prime}=m-m_{s}, \quad k_{s}^{\prime}=k-k_{s}, \quad R_{s, k_{s}}^{+} \equiv R_{m_{s}+k_{s}, k_{s}}^{+}
$$




$$
R_{n-s, k-k_{s}}^{+} \equiv R_{m_{s}^{\prime}+k_{s}^{\prime}, k_{s}^{\prime}}^{+} \equiv R_{n-s, k_{s}^{\prime}}^{+}=R_{s^{\prime}, k_{s}^{\prime}}^{+}
$$

Further, ${ }_{s} y^{\prime}, \gamma_{n-s, k_{s}^{\prime}},\left({ }_{s} y^{\prime}\right)^{\gamma_{n-s, k_{s}^{\prime}}}$ and $d \mu_{n-s, k_{s}^{\prime}}\left({ }_{s} y^{\prime}\right)$ are determined from the equalities

$y=\uparrow\left({ }_{s} y,{ }_{s} y^{\prime}\right) \gamma_{n, k}=\uparrow\left(\gamma_{s, k_{s}}, \gamma_{n-s, k_{s}^{\prime}}\right), y^{\gamma_{n, k}} \equiv y_{m+1}^{m+1} \ldots y_{m+k}^{\gamma_{m+k}}={ }_{s} y^{\gamma_{s, k_{s}}}\left({ }_{s} y^{\prime}\right)^{\gamma_{n-s, k_{s}^{\prime}}}$

and

$$
d \mu_{n, k}(y)=d \mu_{s, k_{s}}\left({ }_{s} y\right) d \mu_{n-s, k_{s}^{\prime}}\left({ }_{s} y^{\prime}\right) .
$$

If $m_{s}>0\left(k_{s}>0\right)$, we assume

$$
\begin{gathered}
\left\{n_{1}, \ldots, n_{s}\right\} \cap\{1, \ldots, m\}=\left\{j_{1}, \ldots, j_{m_{s}}\right\}, \quad j_{1}<\ldots<j_{m_{s}}, \\
\left(\left\{n_{1}, \ldots, n_{s}\right\} \cap\{m+1, \ldots, m+k\}=\left\{m+i_{i}, \ldots, m+i_{k_{s}}\right\}, \quad i_{1}<\ldots<i_{k_{s}}\right) .
\end{gathered}
$$

Then, obviously

$$
{ }_{s} y=\left(y_{j_{1}}, \ldots, y_{j_{m_{s}}}, y_{m+i_{1}}, \ldots, y_{m+i_{k_{s}}}\right)
$$

and

$$
d_{s} y=d y_{j_{1}} \ldots d y_{j_{m_{s}}} d y_{m+i_{1}} \ldots d y_{m+i_{k_{s}}} .
$$

Let $s \in\{1, \ldots, m+k-1\}, m_{s}, k_{s}$ and the coordinates of the point ${ }_{s} y$ be fixed. Then ${ }_{s} y^{\prime}=\left(y_{j_{1}^{\prime}}, \ldots, y_{j_{m_{s}^{\prime}}^{\prime}}, y_{m+i_{1}^{\prime}}, \ldots, y_{m+i_{k_{s}^{\prime}}^{\prime}}\right)$ also is uniquely determined. Assume $y_{i_{1}^{\prime}}=y_{m+i_{1}^{\prime}}$.

When $G \subseteq R_{n, k}^{+}$is a measurable set, and $p \geq 1$,

$$
\begin{aligned}
& L_{p, \gamma_{n, k}}(G)= \\
& \qquad f-\text { izm. : }\left\|K(f): L_{q, \gamma_{n, k}}(G)\right\|=\left(\int_{G}|f(y)|^{p} d \mu_{\gamma_{n, k}}(y)\right)^{1 / p}<+\infty \mid
\end{aligned}
$$

is the space of functions summable in the $p$-th degree on the set $G$.

In the sequel, we repeatedly will use the following easily provable properties of the generalized shift operator $T^{y}=T_{\mu_{n, k}}^{y}[4,5]$ :

T1) the operator $T_{\mu_{n, k}}^{y}$ is self-adjoint

$$
\begin{gathered}
\int_{R_{m+k, k}^{+}} v(s) T_{\gamma_{n, k}}^{s} u(x) d \mu_{\gamma_{n, k}}(s)=\int_{R_{m+k, k}^{+}} u(s) T_{\gamma_{n, k}}^{s} v(x) d \mu_{\gamma_{n, k}}(s), \\
\left.T 2) T^{y} 1=1 ; T 3\right) T^{y}(C f)=C T^{y}(f), C \in R
\end{gathered}
$$


T4) if $|f| \leq|g|$, then $T^{y}(|f|) \leq T^{y}(|g|)$;

T5) if $p>1$, then $\left(\left|T^{y}(f)\right|\right)^{p} \leq T^{y}(|f|)^{p}$;

T6) $\left(\int_{R_{m+k, k}^{+}}\left(T_{\gamma_{n, k}}^{s}(|f(x)|)\right)^{p} d \mu(y)\right)^{1 / p} \leq\left\|f: L_{q, \gamma_{n, k}}\left(R_{n, k}^{+}\right)\right\|$;

T7) $T_{\gamma_{n, k}}^{\left(s y, s y^{\prime}\right)} f\left({ }_{s} x,{ }_{s} x^{\prime}\right)=T_{\gamma_{s, k_{s}}}^{s y}\left(T_{\gamma_{n-s, k_{s}^{\prime}} y^{\prime}}^{{ }^{\prime}} f\left({ }_{s} x,{ }_{s} x^{\prime}\right)\right)=T_{\gamma_{n-s, k_{s}^{\prime}}^{s} y^{\prime}}\left(T_{\gamma_{s, k_{s}}}^{s y} f\left({ }_{s} x,{ }_{s} x^{\prime}\right)\right)$.

We need also the following known inequalities.

The Holder inequality: Let $1<p<\infty, q=\frac{p}{p-1}$ then

$$
\begin{aligned}
& \left|\int_{R_{n, k}^{+}} f_{1}(y) f_{2}(y) d \mu_{\gamma_{n, k}}(y)\right| \\
& \leq\left(\left.\int_{R_{n, k}^{+}}\left|f_{1}(y)\right|^{p} d \mu_{\gamma_{n, k}}(y)\left|\int_{R_{n, k}^{+}}\right| f_{2}(y)\right|^{q} d \mu_{\gamma_{n, k}}(y)\right)^{1 / q},
\end{aligned}
$$

and the existence of the left hand side follows from the existence of the right hand side.

The Minkovsky inequality. Let $1 \leq p<\infty, \mu(x)$ and $\nu(y)$ be RadonStieltjes's non-negative measures on the sets $G$ and $\Omega$, respectively. Then

$$
\left[\int _ { G } \left(\left.\left.\int_{\Omega}|\varphi(x, y)| d \nu(y)\right|^{p} d \mu(x)\right|^{1 / p} \leq \int_{G}\left[\int_{\Omega}|\varphi(x, y)|^{p} d \mu(x)\right]^{1 / p} d \nu(y) .\right.\right.
$$

The Young inequality. Let $1 \leq p, q, r \leq \infty, \frac{1}{q}=\frac{1}{p}+\frac{1}{r}-1$ and

$$
K(f)(x)=\int_{R_{n, k}^{+}} K(x-y) f(y) d \mu_{\gamma_{n, k}}(y)
$$

then

$$
\left\|K(f): L_{q, \gamma_{n, k}}\left(R_{n, k}^{+}\right)\right\| \leq\left\|K: L_{r, \gamma_{n, k}}\left(R_{n, k}^{+}\right)\right\|\left\|f: L_{p, \gamma_{n, k}}\left(R_{n, k}^{+}\right)\right\| .
$$


Hardy-Littlewood theorem on maximum functions. Let $1 \leq p \leq$ $q \leq \infty, \bar{u}(t)$ and $\bar{\nu}(t)$ be functions positive on the interval $(0,+\infty)$. Then for the validity of the inequality

$$
\left(\left.\int_{0}^{\infty}\left|\bar{u}(t) \int_{0}^{\tau} f(t) d \tau\right|^{q} d t\right|^{1 / q} \leq k\left(\int_{0}^{\infty}|f(t) \bar{\nu}(t)|^{p} d t\right)^{1 / p}\right.
$$

of the constant $k$ independent of $f$, it is necessary and sufficient the condition

$$
\sup _{\xi>0}\left(\int_{\xi}^{\infty}|\bar{u}(t)|^{q} d t\right)^{1 / q}\left(\int_{0}^{\xi}|\bar{\nu}(t)|^{-p^{\prime}} d t\right)^{1 / p^{\prime}}<\infty
$$

to be fulfilled.

\section{Basic Results}

The operator $A$ is called subadditive if for any $\lambda, \mu>0$ and any functions $f$ and $g$ from the domain of definition of operator $A$

$$
|A(\lambda f+\mu g)(x)| \leq \lambda|A(f)(x)|+\mu|A(g)(x)|
$$

Definition 1. Let $1 \leq p \leq q<+\infty$. We say that subadditive operator $A$ belongs to the class $\bar{K}_{\gamma_{n, k}}(p, q)$ if $A: L_{p, \gamma_{n, k}}\left(R_{n, k}^{+}\right) \rightarrow L_{q, \gamma_{n, k}}\left(R_{n, k}^{+}\right)$is bounded and for any function $u \in L_{p, \gamma_{n, k}}\left(R_{n, k}^{+}\right)$with compact support,

$$
|A u(x)| \leq c \int_{R_{m+k, k}^{+}}|y|^{-\beta} T_{\gamma_{n, k}}^{y}|u(x)| d \mu_{\gamma_{n, k}}(y), \quad x \notin \sup p u, T_{\gamma_{n, k}}^{y}
$$

where $\beta=\left(m+k+\left|\gamma_{n, k}\right|\right)\left(\left(1 / p^{\prime}\right)+q^{-1}\right)$ and $C$ is independent of $u$.

In the case $p=q$, the operators $A \in \bar{K}_{\gamma_{n, k}}(p, q)$ may be singular. Let us consider

1. The Poisson $B_{\gamma_{n, k}}$ integral:

$$
\left(U_{\gamma_{n, k}} f\right)(x) \stackrel{d f}{=} \sup _{t>0}\left(U_{\gamma_{n, k}} f\right)(x, t)
$$




$$
\left(U_{\gamma_{n, k}} f\right)(x, t)=a_{\nu} \int_{R_{m+k, k}^{+}} t\left(t^{2}+|y|^{2}\right)^{-\frac{m+k+1+\left|\gamma_{n, k}\right|}{2}} f(y) d \mu_{\gamma_{n, k}}(s) \text {; }
$$

2. $B_{\gamma_{n, k}}$ - maximum function:

$$
\begin{gathered}
M_{\gamma_{n, k}} f(x)=\sup _{r>0}|B(0, r)|_{\gamma_{n, k}}^{-1} \int_{B(0, r)} T_{\gamma_{n, k}}^{y}|f(x)| d \mu_{\gamma_{n, k}}(y), \\
B(0, r)=\left\{y \in R_{m+k, k}^{+}:|y|<\varepsilon\right\}, \quad|B(0, r)|_{\gamma_{n, k}}=\int_{B(0, r)} d \mu_{\gamma_{n, k}}(y) ;
\end{gathered}
$$

3. $B_{\gamma_{n, k}}$ - fractional maximum function:

$$
M_{\gamma_{n, k}}^{\alpha} f(x)=\sup _{r>0}|B(0, r)|_{\gamma_{n, k}}^{\frac{\alpha}{m+k+\gamma_{n, k}}-1} \int_{B(0, r)} T_{\gamma_{n, k}}^{y}|f(x)| d \mu_{\gamma_{n, k}}(y)
$$

4. $B_{m+k, 2 \nu}$ Riesz potential

$$
I_{\gamma_{n, k}}^{\alpha} f(x)=\int_{R_{m+k, k}^{+}} T^{y}|x|^{\alpha-(m+k)-\left|\gamma_{n, k}\right|} f(y) d \mu_{\gamma_{n, k}}(y) .
$$

It is known that [5] $U_{\gamma_{n, k}}, M_{\gamma_{n, k}} \in \bar{K}_{\gamma_{n, k}}(p, p)$ if $p>1$ and $M_{\gamma_{n, k}}^{\alpha}, I_{\gamma_{n, k}}^{\alpha} \in \bar{K}_{\gamma_{n, k}}(p, p)$ for $1<p<q<\infty$ and $\alpha=\left(m+k+\left|\gamma_{n, k}\right|\right)\left(\frac{1}{p}-\frac{1}{q}\right)$.

In the sequel, $C$ is a positive number different in various inequalities.

When $s \in\{1, \ldots, m+k\}, A_{p, \gamma_{n, k}}\left({ }_{s} x\right)$ denotes a union of all functions measurable on the set $R_{m+k, k}^{+}$and belonging to $L_{p, \gamma_{n, k}}\left(\left\{x \in R_{m+k, k}^{+}:\left|{ }_{s} x\right| \geq \xi\right\}\right)$, for each number $\xi>0$.

Further, $\alpha_{p, s}=\left(s+\left|\gamma_{k_{s}}\right|\right) / p^{\prime}$.

For the functions $u \in A_{p, \gamma_{n, k}}\left({ }_{s} x\right)$ we introduce the characteristics

$$
\Omega_{p, \mu_{n, k}}^{\left(s^{x}\right)}(u, \xi)=\left\{\int_{\left\{x \in R_{m+k, k}^{+}:\left.\right|_{s} x \mid \geq \xi\right\}}|u(x)|^{p} d \mu_{\gamma_{n, k}}(x)\right\}^{1 / p}, \xi>0,
$$


and the set

$$
J_{p, \mu_{n, k}}\left({ }_{s} x\right)=\left\{u \in A_{p, \gamma_{n, k}}\left({ }_{s} x\right): \int_{0}^{\xi} t^{\alpha_{p, s}-1} \Omega_{p, \mu_{n, k}}^{\left({ }_{s} x\right)}(u, t) d t<+\infty, \quad \forall \xi>0\right\} .
$$

By definition, the non-negative function $\alpha(t), 0<t<\infty$ belongs to the set $N$ if for small $\varepsilon>0$ for almost all $t \in(0, \varepsilon) \alpha(t)>0$ and $\forall \varepsilon>0$ the integral $\int_{0}^{\varepsilon} \alpha(t) d t$ converges.

Let $1 \leq p<\infty$ and $\varphi \in N$. Let us introduce the space

$$
I_{p, \mu_{n, k}}^{\left(s_{s} x\right)}(\varphi)=\left\{u-i z m .\left\|u: I_{p, \mu_{n, k}}^{\left({ }_{s} x\right)}(\varphi)\right\|^{p} \stackrel{d f}{=} \int_{0}^{\infty}\left(\Omega_{p, \mu_{n, k}}^{(s x)}(u, \xi)\right)^{p} \varphi(\xi) d \xi<\infty\right\}
$$

Let

$$
\begin{aligned}
& L_{p, \gamma_{n, k}} \\
& =\left[f-i z m .:\left\|f: L_{p, \gamma_{n, k}}(G)\right\|=\left(\int_{G}|f(y) \omega(y)|^{p} d \mu_{\gamma_{n, k}}(y)\right)^{1 / p}<+\infty\right]
\end{aligned}
$$

be the $L_{p, \gamma_{n, k}}(G)$ space with the weight $\omega(y)$.

The main results of the paper are in the following three theorems.

Theorem 1. Let $A \in \bar{K}_{\gamma_{n, k}}(p, q), s \in\{1, \ldots, m+k\}$ and $u \in J_{p, \mu_{n, k}}\left({ }_{s} x\right)$. Then for almost all $x \in R_{m+k, k}^{+}$there exists $v(x)=A(u)(x)$ and it holds the estimation

$$
\Omega_{q, \mu_{n, k}}^{\left({ }_{s} x\right)}(v, \xi) \leq c \xi^{-\alpha_{p, s}} \int_{0}^{\xi} t^{\alpha_{p, s}-1} \Omega_{p, \mu_{n, k}}^{\left({ }_{s} x\right)}(u, t) d t, \quad \xi>0
$$

where the constant $c$ is independent of $u$ and $\xi$.

Theorem 2. Let $1<p \leq q<\infty, A \in \bar{K}_{\gamma_{n, k}}(p, q), \varphi, \psi \in N, s \in$ $\{1, \ldots, m+k\}$ and the following condition be fulfilled

$$
\sup _{t>0}\left(\int_{0}^{\infty}\left|\xi^{-\alpha_{p, s}} \psi^{\frac{1}{q}}(\xi)\right|^{q}\right)^{1 / q}\left(\int_{0}^{\infty}\left|\varphi^{1 / p}(\xi) \xi^{\left(1-\alpha_{p, s}\right)}\right|^{-p^{\prime}}\right)^{1 / p^{\prime}}<\infty . \quad(\varphi, \psi)
$$


Then operator $A$ acts from the space $I_{p, \mu_{n, k}}^{(s x)}$ to $I_{q, \mu_{n, k}}^{(s x)}(\psi)$ and it holds the following inequality

$$
\left\|A u: I_{q, \mu_{n, k}}^{\left({ }_{s} x\right)}(\psi)\right\| \leq c\left\|A u: I_{p, \mu_{n, k}}^{(s x)}(\varphi)\right\|,
$$

where $c$ is independent of the function $u$.

Theorem 3. Let $1<p \leq q<\infty, A \in \bar{K}_{\gamma_{n, k}}(p, q), s \in\{1, \ldots, m+k\}, \omega(t)$ and $\omega_{1}(t)$ be non-negative and nondecreasing functions on the interval $(0,+\infty)$ such that

$$
c^{-1} \omega^{p}(\xi) \leq \int_{0}^{\xi} \omega^{p}(t) t^{-1} d t \leq c \omega^{p}(\xi)
$$

and

$$
c_{1}^{-1} \omega_{1}^{q}(\xi) \leq \int_{0}^{\xi} \omega_{1}^{q}(t) t^{-1} d t \leq c_{1} \omega_{1}^{p}(\xi) .
$$

Then if the condition

$$
\sup _{t>0}\left(\int_{t}^{\infty}\left(\xi^{-\alpha_{p, s}} \omega_{1}(\xi)\right)^{q} \frac{d \xi}{\xi}\right)^{1 / q}\left(\int_{0}^{\infty}\left(\omega(\xi) \xi^{-\alpha_{p, s}}\right)^{-p^{\prime}} \frac{d \xi}{\xi}\right)^{1 / p^{\prime}}<\infty
$$

is fulfilled, $L_{q, \gamma_{n, k}}\left(\omega\left(\left|{ }_{s} x\right|\right) ; R_{n, k}^{+}\right)$and it holds the inequality

$$
\left\|A u: L_{q, \gamma_{n, k}}\left(\omega\left(||_{s} x \mid\right) ; R_{n, k}^{+}\right)\right\| \leq c\left\|A u: L_{p, \gamma_{n, k}}\left(\omega\left(\left.\right|_{s} x \mid\right) ; R_{n, k}^{+}\right)\right\|
$$

where $c$ is independent of the function $u$.

\section{Pointwise and Integral Inequalities in the Terms of $\Omega_{p, \mu_{n, k}}^{\left({ }_{s}\right)}$ Characteristics}

Let $\chi_{E}(t)$ be a characteristic function of the set $E \subset[0,+\infty)$, and $s \in$ $\{1, \ldots, m+k-1\}$. Assume

$$
\begin{gathered}
u_{\xi, s}(s)=\chi_{[0, \xi]}\left(\left|{ }_{s} y\right|\right)|u(y)|, \quad y \in R_{n, k}^{+}, \quad \xi>0 ; \\
u_{\xi, s}^{0}\left({ }_{s} y\right)=\left(\int_{R_{s, k_{s}^{\prime}}^{+}} u_{\xi, s}^{p}\left({ }_{s} y, s y^{\prime}\right) d \mu_{s^{\prime}, k_{s}^{\prime}}\left({ }_{s} y^{\prime}\right)\right)^{1 / p}, \quad, \quad y \in R_{m_{s}+k_{s}, k_{s}}^{+}
\end{gathered}
$$




$$
I_{\beta, s}(u, \xi)(x)=\int_{R_{m+k, k}^{+}} \frac{u_{\xi, s}(y) d \mu_{n, k}(y)}{\left(\left|{ }_{s} x^{\prime}-{ }_{s} y^{\prime}\right|+\left.\right|_{s} x \mid+\xi\right)^{\beta}}, \quad x \in R_{n, k}^{+} .
$$

And also, if $s=m+k$, we assume

$$
\begin{gathered}
u_{\xi, s}^{0}\left({ }_{s} y\right)=u_{\xi, s}(y) \equiv \chi_{[0, \xi]}(|y|)|u(y)|, \quad y \in R_{n, k}^{+}, \quad \xi>0 ; \\
I_{\beta, s}(u, \xi)(x)=(|x|+\xi)^{-\beta} \int_{\left\{R_{m+k, k}^{+}:|y| \leq \xi\right\}} u(y) d \mu_{n, k}(y), \quad x \in R_{n, k}^{+} .
\end{gathered}
$$

Everywhere in the sequel, $\sigma_{s}=m_{s}-\beta p^{\prime}$.

Lemma 1. Let $s \in\{1, \ldots, m+k\}, 1<p \leq q<+\infty$ and $u \in A_{p, \gamma_{n, k}}\left({ }_{s} x\right)$, $\beta=\left(m+k+\left|\gamma_{n, k}\right|\right)\left(\left(1 / p^{\prime}\right)+q^{-1}\right)$ and $\xi>0$. Then

a) $\forall x \in\left\{x \in R_{m+k, k}^{+}:\left|{ }_{s} x\right| \geq \xi\right\}$,

$I_{\beta, s}(u, \xi)(x) \leq$

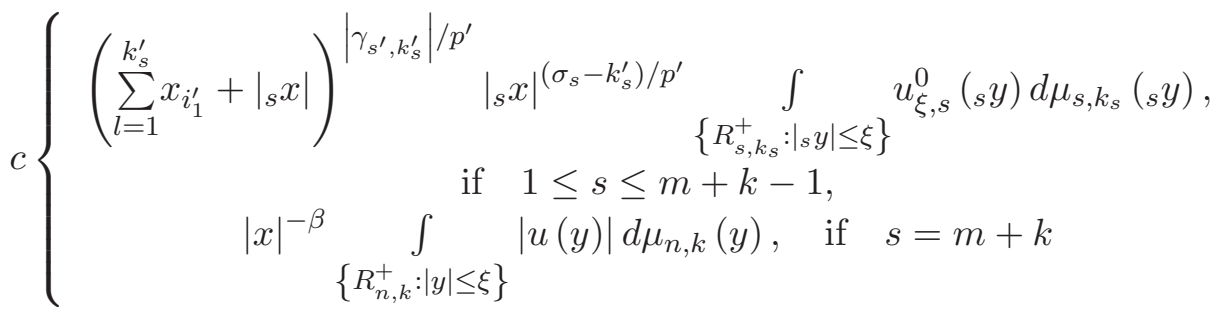

b) $\left\|I_{\beta, s}(u, \xi): L_{p, \gamma_{n, k}}\left(R_{n, k}^{+}\right)\right\| \leq c \xi^{-\alpha_{p, s}} \quad \int \quad u_{\xi, s}^{0}\left({ }_{s} y\right) d \mu_{s, k_{s}}\left({ }_{s} y\right)$,

$$
\left\{R_{s, k_{s}}^{+}:\left|s_{s} y\right| \leq \xi\right\}
$$

where the constant $c$ is independent of $u$ and $\xi$.

Lemma 2. Let $s \in\{1, \ldots, m+k\}$ and $u \in J_{p, \mu_{n, k}}\left({ }_{s} x\right)$, then

$$
\int_{R_{m_{s}+k_{s}, k_{s}}^{+}} u_{\xi, s}^{0}\left({ }_{s} y\right) d \mu_{s, k_{s}}\left({ }_{s} y\right) \leq c \int_{0}^{\xi} t^{\alpha_{p, s}-1} \Omega_{p, \mu_{n, k}}^{(s, x)}(u, t) d t
$$

where the constant $c$ is independent of $u$ and $\xi$.

Proof of Lemmas. Let us prove point a) of lemma 1. Let $s \in\{1, \ldots$, $m+k-1\}$. Introduce the denotation $d \mu\left(y_{i_{l}^{\prime}}\right)=\left(y_{i_{l}^{\prime}}\right)^{\gamma_{i j}} d y_{i_{j}^{\prime}}, \quad l=1, \ldots, k_{s}^{\prime} ; a_{j}=\left(\sum_{l=1}^{j}\left|x_{i_{l}^{\prime}}-y_{i_{l}^{\prime}}\right|+\left|{ }_{s} x\right|\right), j=1, \ldots, k_{s}^{\prime}$. 
Successive application of the estimation

$$
\begin{aligned}
& \Lambda=\int_{R^{+}}\left(a_{j}+d\right)^{\sigma} d \mu\left(y_{i_{l}^{\prime}}\right) \\
& \quad \leq c\left(\left(x_{i_{l}^{\prime}}\right)^{\gamma_{i_{l}^{\prime}}}\left(d+a_{i-1}\right)^{\sigma+1}+\left(d+x_{i_{j}^{\prime}}+a_{i-1}\right)^{\sigma+\gamma_{i_{j}^{\prime}}}{ }^{1}\right)
\end{aligned}
$$

where $d>0, j \geq 1$, leads to the proof of the inequality

$$
\begin{aligned}
& B=\left\{\int_{R_{m_{s}^{\prime}+k_{s}^{\prime}, k_{s}^{\prime}}} \frac{d \mu_{s^{\prime}, k_{s}^{\prime}}\left({ }_{s} y^{\prime}\right)}{\left(\left|{ }_{s} x^{\prime}-{ }_{s} y^{\prime}\right|+\left|{ }_{s} x\right|\right)^{\beta_{p^{\prime}}}}\right\}^{1 / p^{\prime}} \leq \\
& \leq c\left(\sum_{l=1}^{k_{s}^{\prime}} x_{i_{l}^{\prime}}+{ }_{s} x \mid \sum_{l=1}^{\left.k_{s}^{\prime} \gamma_{i_{l}^{\prime}}\right)^{/ p^{\prime}}\left|{ }_{s} x\right|^{\left(\sigma-k_{s}^{\prime}\right) / p^{\prime}} .}\right.
\end{aligned}
$$

Applying the Foubini theorem, and then the Holder inequality in the inner integral, we get

$$
\begin{aligned}
& I_{\beta, s}(u, \xi)(x)=\int_{R_{m+k, k}^{+}} \frac{u_{\xi, s}(y) d \mu_{n, k}(y)}{\left(\left|{ }_{s} x^{\prime}-{ }_{s} y^{\prime}\right|+\left|{ }_{s} x\right|\right)^{\beta}}= \\
& =\iint_{R_{m_{s}+k_{s}, k_{s}}^{+}}\left\{\frac{u_{\xi, s}(y) d \mu_{s^{\prime}, k_{s}^{\prime}}\left({ }_{s} y^{\prime}\right)}{\left(\left|{ }_{s} x^{\prime}-{ }_{s} y^{\prime}\right|+{ }_{s} x \mid\right)^{\beta}}\right\} d \mu_{s}, k_{s}\left({ }_{s} y\right) \leq \\
& \leq \int_{R_{m_{s}+k_{s}, k_{s}}^{+}}\left\{\int_{R_{m_{s}^{\prime}+k_{s}^{\prime}, k_{s}^{\prime}}^{+}} \frac{d \mu_{s^{\prime}, k_{s}^{\prime}}\left({ }_{s} y^{\prime}\right)}{\left(\left|x_{s} x^{\prime} y^{\prime}\right|+\left|{ }_{s} x\right|\right)^{\beta p^{\prime}}}\right\}^{1 / p^{\prime}} \times \\
& \times\left(\int_{R_{m_{s}^{+}+k_{s}^{\prime}, k_{s}^{\prime}}} u_{\xi, s}^{p}\left({ }_{s} y,{ }_{s} y^{\prime}\right) d \mu_{s^{\prime}, k_{s}^{\prime}}\left({ }_{s} y^{\prime}\right)\right)^{1 / p} d \mu_{s, k_{s}}\left({ }_{s} y\right)=
\end{aligned}
$$




$$
=B \int_{R_{m_{s}+k_{s}, k_{s}}^{+}} u_{\xi, s}^{0}\left({ }_{s} y\right) d \mu_{s, k_{s}}\left({ }_{s} y\right) .
$$

Allowing for estimation $B$, point a) of lemma 1 , in the case $s \in\{1, \ldots$, $m+k-1\}$ was proved. While the case $s=m+k$ directly follows from the definition $I_{\beta, m+k}(u, \xi)$.

Now let us prove point b) of lemma 1 in the case $s \in\{1, \ldots, m+k-1\}$. First of all, note that having applied the Young inequality, where $r>1, r^{-1}=$ $\left(p^{\prime}\right)^{-1}+q^{-1}$, we get

$$
\begin{gathered}
A=\left(\int _ { R _ { m _ { s } + k _ { s } , k _ { s } } } \left(\int_{R_{m_{s}^{\prime}+k_{s}^{\prime}, k_{s}^{\prime}}} \frac{u_{\xi, s}(y) d \mu_{s^{\prime}, k_{s}^{\prime}}\left({ }_{s} y^{\prime}\right)}{\left(\left|{ }_{s} x^{\prime}-{ }_{s} y^{\prime}\right|+\left|{ }_{s} x\right|+\xi\right)^{\beta}}\left|d \mu_{s^{\prime}, k_{s}^{\prime}}\left({ }_{s} x^{\prime}\right)\right|^{1 / q} \leq\right.\right. \\
\leq c\left(\int_{R_{m_{s}^{\prime}+k_{s}^{\prime}, k_{s}^{\prime}}} \frac{d \mu_{s^{\prime}, k_{s}^{\prime}}\left({ }_{s} y^{\prime}\right)}{\left(\left|y_{s}^{\prime}\right|+\left|{ }_{s} x\right|+\xi\right)^{\beta r}} u_{\xi, s}^{0}\left({ }_{s} y\right) \leq\right. \\
\leq c\left(\left|{ }_{s} x\right|+\xi\right)^{\frac{\left|\gamma_{s^{\prime}, k_{s}^{\prime}}\right|+s^{\prime}}{r}-\beta} u_{\xi, s}^{0}\left({ }_{s} y\right)=A^{\prime} .
\end{gathered}
$$

Having applied the Foubini theorem on reduction of multiple integral to interated integral and using the transition

$$
\begin{aligned}
& \left.\left(\iint_{1}\left\{\iint_{1^{\prime}}(\ldots)\right\}^{\prime}\right\}^{q / q}=\left(\int\left[\int\left\{\int_{1} \int_{1^{\prime}}(\ldots)\right\}_{2^{\prime}}\right\}^{(1 / q) q}\right)^{1 / q}\right)^{\leq} \\
& =\iint\left[\int_{1}\left\{\int_{1^{\prime}}\left(\int_{2^{\prime}}(\ldots)\right\}^{q}\right\}^{1 / q}\right)^{q 1 / q}
\end{aligned}
$$

and then taking into account the last estimation, we get

$$
\left\|I_{\beta, s}(u, \xi): L_{p, \gamma_{n, k}}\left(R_{n, k}^{+}\right)\right\|=\left(\int_{R_{m_{s}+k_{s}, k_{s}}^{+}} d \mu_{s, k_{s}}\left({ }_{s} x\right) \int{ }_{R_{m_{s}^{\prime}+k_{s}^{\prime}, k_{s}^{\prime}}^{+}} d \mu_{s^{\prime}, k_{s}^{\prime}}\left({ }_{s} x^{\prime}\right) \times\right.
$$




$$
\begin{aligned}
& \times\left(\int d \mu_{s, k_{s}}\left({ }_{s} y\right) \int_{R_{m_{s}+k_{s}, k_{s}}^{+}} \int_{m_{s}^{\prime}+k_{s}^{\prime}, k_{s}^{\prime}} \frac{u_{\xi, s}(y) d \mu_{s^{\prime}, k_{s}^{\prime}}\left({ }_{s} y^{\prime}\right)}{\left(\left|{ }_{s} x^{\prime}-{ }_{s} y^{\prime}\right|+\left|{ }_{s} x\right|\right)^{\beta}}\right)^{1 / q} \leq \\
& \leq c\left(\int_{R_{m_{s}+k_{s}, k_{s}}^{+}}\left(\int_{R_{m_{s}+k_{s}, k_{s}}^{+}} A d \mu_{s, k_{s}}\left({ }_{s} y\right)\right)^{1 / q} d \mu_{s, k_{s}}\left({ }_{s} x\right)\right)^{1 / q} \leq \\
& \leq c\left(\int_{R_{m_{s}+k_{s}, k_{s}}^{+}}\left(\int_{R_{m_{s}+k_{s}, k_{s}}^{+}} A^{\prime} d \mu_{s, k_{s}}\left({ }_{s} y\right)\right)^{1 / q} d \mu_{s, k_{s}}\left({ }_{s} x\right)\right)^{1 / 2}= \\
& c\left(\left.\int_{R_{m_{s}+k_{s}, k_{s}}^{+}}\left(\left(\left|{ }_{s} x\right|+\xi\right)^{\frac{\left|\gamma_{k_{s}}\right|+s^{\prime}}{r}-\beta}\right)^{q} d \mu_{s, k_{s}}\left({ }_{s} x\right)\right|_{R_{m_{s}+k_{s}, k_{s}}^{+}} ^{1 / q} u_{\xi, s}^{0}\left({ }_{s} y\right) d \mu_{s, k_{s}}\left({ }_{s} y\right)\right. \\
& \leq c \xi^{-\alpha_{p, s}} \quad \int \quad u_{\xi, s}^{0}\left({ }_{s} y\right) d \mu_{s, k_{s}}\left({ }_{s} y\right) . \\
& R_{m_{s}+k_{s}, k_{s}}^{+}
\end{aligned}
$$

This proves point b) of lemma 1 in the case $s \in\{1, \ldots, m+k-1\}$.

Now let $s=m+k$. For further reasonings we need transition to spherical coordinates.

Let $S_{n, k}^{+}=\left\{x \in R_{n, k}^{+}:|x|=1\right\}$ - unit sphere $R_{n, k}^{+}$. Then passing to spherical coordinates centered at the origin of coordinates $\left(y \rightarrow(r, \theta), \theta \in S_{n, k}^{+}, r \geq 0\right)$, wed get

$$
d \mu_{n, k}(y)=y^{\gamma_{n, k}} d y=\left(\frac{y}{|y|}\right)^{\gamma_{n, k}} d y=\theta^{\gamma_{n, k}} r^{n-1+\left|\gamma_{n, k}\right|} d r d \sigma(\theta),
$$

where $d \sigma(\theta)$ is an element of the surface of the sphere $S_{n, k}^{+}$.

Then, allowing for the estimation

$$
B=\left(\int_{R_{m+k, k}^{+}} \frac{d \mu_{n, k}(x)}{(|x|+\xi)^{\beta q}}\right)^{1 / q}=\left(\int_{S_{n, k}^{+}} \theta^{\gamma_{n, k}} d \sigma(\theta) \int_{0}^{\infty} \frac{r^{m+k+\left|\gamma_{n, k}\right|-1} d r}{(r+\xi)^{\beta q}}\right)^{1 / q} \leq
$$




$$
\leq c\left(\int_{0}^{\infty} \frac{r^{m+k+\left|\gamma_{n, k}\right|-1} d r}{(r+\xi)^{\beta q}}\right)^{1 / q} \leq c \xi^{\left(m+k+\left|\gamma_{n, k}\right|-\beta q\right) / q}=c \xi^{-\alpha_{p, m+k}}
$$

we get

$$
\begin{gathered}
\left\|I_{\beta, s}(u, \xi): L_{p, \gamma_{n, k}}\left(R_{n, k}^{+}\right)\right\|= \\
=B \int_{\left\{R_{m+k, k}^{+}:|y|<\xi \mid\right\}}|u(y)| d \mu_{n, k}(y) \leq c \xi^{-\alpha_{p, m+k}} \int_{\left\{R_{m+k, k}^{+}:|y|<\xi \mid\right\}}|u(y)| d \mu_{n, k}(y) .
\end{gathered}
$$

This proves point $\mathrm{b}$ ) in the case $s=m+k$, and Lemma 1. Now prove lemma 2.

Let $s \in\{1, \ldots, m+k-1\}$. Assume

$$
\begin{aligned}
& \bar{u}_{\xi, s}\left({ }_{s} y\right)=\left(\left.\int_{R_{s^{\prime}, k_{s}^{\prime}}^{+}}\left|u\left({ }_{s} y,,_{s} y^{\prime}\right)\right|^{p} d \mu_{s^{\prime}, k_{s}^{\prime}}\left({ }_{s} y^{\prime}\right)\right|^{1 / p},\right. \\
& N=\int_{t}^{\xi} \bar{u}_{\xi, s}(r \theta) r^{\left(s-1+\left|\gamma_{s, k_{s}}\right|\right) / p} \frac{d r}{r^{\alpha+1-\left(s-1+\left|\gamma_{s, k_{s}}\right|\right) / i^{\prime}}} .
\end{aligned}
$$

Applying the Holder inequality, we get

$$
\begin{gathered}
N \leq\left(\int_{t}^{\xi} \bar{u}_{\xi, s}^{p}(r \theta) r^{\left(s-1+\left|\gamma_{s, k_{s}}\right|\right)} d r{ }^{1 / p}\left(\int_{t}^{\xi} \frac{d r}{r^{(\alpha+1) p^{\prime}-\left(s-1+\left|\gamma_{s, k_{s}}\right|\right)}}\right)^{1 / p^{\prime}} \leq\right. \\
\leq c\left(\int_{t}^{\xi} \bar{u}_{\xi, s}^{p}(r \theta) r^{\left(s-1+\left|\gamma_{s, k_{s}}\right|\right)} d r\right) t^{\frac{s+\left|\gamma_{s, k_{s} \mid}\right|}{p^{\prime}}(\alpha+1)} .
\end{gathered}
$$

And also, passing to spherical coordinates, using the Dirichlet formula, we get

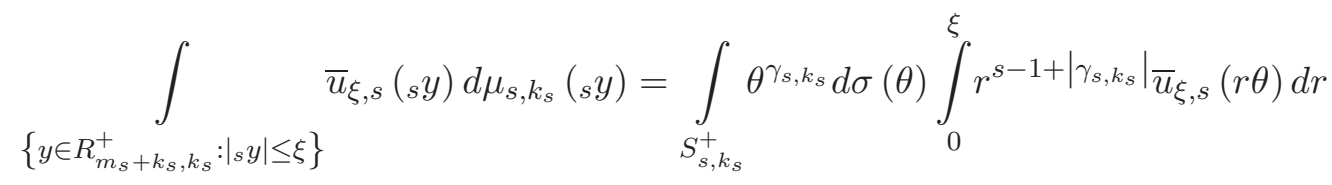




$$
\begin{aligned}
& =(\alpha+1) \int_{S_{s, k_{s}}^{+}} \theta^{\gamma_{s, k_{s}}} d \sigma(\theta) \int_{0}^{\xi} \bar{u}_{\xi, s}(r \theta) r^{s-1+\left|\gamma_{s, k_{s}}\right|}\left(\frac{1}{r^{\alpha+1}} \int_{0}^{r} t^{\alpha} d t\right) d r= \\
& =c \int_{S_{s, k_{s}}^{+}} \theta^{\gamma_{s, k_{s}}} d \sigma(\theta) \int_{0}^{\xi} t^{\alpha} N d t \leq \\
& \leq c \int_{S_{s, k_{s}}^{+}} \theta^{\gamma_{s, k_{s}}} d \sigma(\theta) \int_{0}^{\xi} t^{\alpha}\left(\int_{t}^{\xi} \bar{u}_{\xi, s}^{p}(r \theta) r^{\left(s-1+\left|\gamma_{s, k_{s}}\right|\right)} d r\right) t^{\frac{s+\left|\gamma_{s, k_{s}}\right|}{p^{\prime}}-(\alpha+1)} d t= \\
& =c \int_{S_{s, k_{s}}^{+}} \theta^{\gamma_{s, k_{s}}} d \sigma(\theta) \int_{0}^{\xi}\left(\int_{t}^{\xi} \bar{u}_{\xi, s}^{p}(r \theta) r^{\left(s-1+\left|\gamma_{s, k_{s}}\right|\right)} d r\right) t^{\frac{s+\left|\gamma_{s, k_{s}}\right|}{p^{\prime}}} d t= \\
& =c \int_{0}^{\xi} t^{\frac{s+\mid \gamma_{s, k_{s} \mid}}{p^{\prime}}-1}\left(\int_{S_{s, k_{s}}^{+}} \theta^{\gamma_{s, k_{s}}} d \sigma(\theta)\left(\int_{t}^{\xi} \bar{u}_{\xi, s}^{p}(r \theta) r^{\left(s-1+\left|\gamma_{s, k_{s}}\right|\right)} d r\right)\right)^{1 / p} d t .
\end{aligned}
$$

Using the above obtained formulas, we get

$$
\begin{aligned}
& \int u_{\xi, s}^{0}\left({ }_{s} y\right) d \mu_{s, k_{s}}\left({ }_{s} y\right)= \\
& R_{m_{s}+k_{s}, k_{s}}^{+} \\
& =\int_{R_{m_{s}+k_{s}, k_{s}}^{+}}\left(\int_{R_{s^{\prime}, k_{s}^{\prime}}^{+}} u_{\xi, s}^{p}\left({ }_{s} y,{ }_{s} y^{\prime}\right) d \mu_{s^{\prime}, k_{s}^{\prime}}\left({ }_{s} y^{\prime}\right)\right)^{1 / p} d \mu_{s, k_{s}}\left({ }_{s} y\right)= \\
& =\int_{\left\{y \in R_{m_{s}+k_{s}, k_{s}}:||_{s} y \mid \leq \xi\right\}} \bar{u}_{\xi, s}\left({ }_{s} y\right) d \mu_{s, k_{s}}\left({ }_{s} y\right) \leq \\
& \leq c \int_{0}^{\xi} t^{\alpha}{ }_{p, s^{-1}}\left(\int_{S_{s, k_{s}}^{+}} \theta^{\gamma_{s, k_{s}}} d \sigma(\theta)\left(\int_{t}^{\xi} \bar{u}_{\xi, s}^{p}(r \theta) r^{\left(s-1+\left|\gamma_{s, k_{s}}\right|\right)} d r\right)^{1 / p}\right) d t \leq
\end{aligned}
$$




$$
\begin{aligned}
& \leq c \int_{0}^{\xi} t^{\alpha_{p, s^{-1}}}\left\{\left(\int_{S_{s, k_{s}}^{+}} \theta^{\gamma_{s, k_{s}}} d \sigma(\theta) \int_{t}^{\xi} \bar{u}_{\xi, s}^{p}(r \theta) r^{\left(s-1+\left|\gamma_{s, k_{s}}\right|\right)} d r\right)^{1 / p} \times\right. \\
& \times\left(\left.\int_{S_{s, k_{s}}^{+}} \theta^{\gamma_{s, k_{s}}} d \sigma(\theta)\right|^{1 / p^{\prime}}\right\} t \leq c \int_{0}^{\xi} t^{\alpha_{p, s^{-1}}} \times \\
& \times\left(\int_{\left\{y \in R_{m_{s}+k_{s}, k_{s}}^{+}: t \leq\left.\right|_{s} y \mid \leq \xi\right\}}\left(\int_{R_{s^{\prime}, k_{s}^{\prime}}^{+}}\left|u\left({ }_{s} y,{ }_{s} y^{\prime}\right)\right|^{p} d \mu_{s^{\prime}, k_{s}^{\prime}}\left({ }_{s} y^{\prime}\right)\right)\right)^{1 / p} \leq \\
& \leq c \int_{0}^{\xi} t^{\alpha}{ }_{p, s^{-1}} \Omega_{p, s}(u, t) d t .
\end{aligned}
$$

Lemma 2 , in the case $s \in\{1, \ldots, m+k-1\}$ is proved.

Now let $s=m+k$. Then passing to spherical coordinates, we have

$$
\begin{aligned}
& \int_{\left\{R_{m+k, k}^{+}:|y| \leq \xi\right\}}|u(y)| d \mu_{n, k}(y)=\int_{S_{s, k}^{+}} \theta^{\gamma_{n, k}} d \sigma(\theta) \int_{0}^{\xi}|u(r \theta)| r^{m+k+\left|\gamma_{n, k}\right|-1} d r= \\
& =c \int_{\Omega} d \theta_{s, m_{s}} \int_{0}^{\xi} u(r \theta) r^{m+k+\left|\gamma_{n, k}\right|-1}\left(r^{-(\alpha+1)} \int_{0}^{r} t^{\alpha} d t\right) d r= \\
& =\int_{0}^{\xi} t^{\alpha}\left(\int_{S_{s, k}^{+}} \theta^{\gamma_{n, k}} d \sigma(\theta)\left[\int_{0}^{\xi} u(r \theta) r^{\left(m+k+\left|\gamma_{n, k}\right|-1\right) / p} \frac{d r}{r^{\alpha+1-\left(m+k+\left|\gamma_{n, k}\right|-1\right) / p^{\prime}}}\right]\right) d t \leq \\
& \leq c \int_{0}^{\xi} t^{\alpha}\left[\left.\int_{0}^{\xi} \frac{d r}{r^{(\alpha+1)-\left(m+k+\left|\gamma_{n, k}\right|-1\right)}}\right|^{1 / p^{\prime}} \times\right. \\
& \times\left(\int_{S_{s, k}^{+}} \theta^{\gamma_{n, k}} d \sigma(\theta)\left[\int_{0}^{\xi} u^{p}(r \theta) r^{\left(m+k+\left|\gamma_{n, k}\right|-1\right)} d r \mid\right)^{1 / p}\right] d t \leq
\end{aligned}
$$




$$
\begin{gathered}
\leq c \int_{0}^{\xi} t^{\alpha} \frac{d t}{t^{(\alpha+1)-\left(m+k+\left|\gamma_{n, k}\right|\right) 1 / p^{\prime}}} \times \\
\times\left(\int_{S_{s, k}^{+}} \theta^{\gamma_{n, k}} d \sigma(\theta)\left[\int_{0}^{\xi} u^{p}(r \theta) r^{\left(m+k+\left|\gamma_{n, k}\right|-1\right)} d r \mid\right]\right)^{1 / p} \times \\
\times\left(\int_{S_{s, k}^{+}} \theta^{\gamma_{n, k}} d \sigma(\theta) \int_{0}^{\xi} d t \leq c t^{\left(m+k+\left|\gamma_{n, k}\right|\right)^{1 / p^{\prime}}-1} \Omega_{p}(u, t) d t\right.
\end{gathered}
$$

This completely proves lemma 2 .

\section{Proof of Main Theorems}

Let us take an arbitrary fixed $\xi \in(0, \infty)$ and represent the function $u(y)$ in the form of the sum $u=u_{\xi, s}(y)+\bar{u}_{\xi, s}(y)$, where $\bar{u}_{\xi, s}(y)=u(y)-u_{\xi, s}(y)$. Obviously, $\bar{u}_{\xi, s}(y)=\chi_{[\xi,+\infty)}\left(\left.\right|_{s} y \mid\right) u(y)$. Then $\bar{u}_{\xi, s}(y) \in L_{p, y}\left(R_{m+k, k}^{+}\right)$, and $A \bar{u}_{\xi, s}(x)$ exists almost for all $x \in R_{m+k, k}^{+}$. Now let us prove that $A u_{\xi, s}(x)$ converges for all points $x \in\left\{R_{m+k, k}^{+}:\left|{ }_{s} x\right|>\xi\right\}$. Note that if $\beta>0$, then $T^{y}\left(|x|^{-\beta}\right) \leq c|x-y|^{-\beta}$, and furthermore, if $x \in\left\{R_{m+k, k}^{+}:\left|{ }_{s} x\right|>\xi\right\}$ and $|s y| \leq \frac{\xi}{2}$, then

$$
c\left(\left|{ }_{s} x^{\prime}-{ }_{s} y^{\prime}\right|+\left|{ }_{s} x\right|+\xi\right)^{-\beta} \leq|x-y|^{-\beta} \leq c_{1}\left(\left|{ }_{s} x^{\prime}-{ }_{s} y^{\prime}\right|+\left|{ }_{s} x\right|+\xi\right)^{-\beta} .
$$

Taking this condition $A \in \bar{K}_{\gamma_{n, k}}(p, q)$ and self-adjointness of the operator $T^{y}$ into account, by point a) of lemma 1 we get

$$
\begin{gathered}
\left|A\left(u_{\xi, s}(x)\right)\right| \leq \int_{R_{m+k, k}^{+}}|y|^{-\beta} T_{\mu_{n, k}}^{y}\left(\left|u_{\xi, s}(x)\right|\right) d \mu_{\gamma_{n, k}}(y)= \\
=\int_{R_{m+k, k}^{+}}\left|u_{\xi, s}(y)\right| T_{\mu_{\gamma_{n, k}}}^{y}\left(|x|^{-\beta}\right) d \mu_{\gamma_{n, k}}(y) \leq c \int_{R_{m+k, k}^{+}}\left|u_{\xi, s}(y)\right||x-y|^{-\beta} d \mu_{\gamma_{n, k}}(y) \leq
\end{gathered}
$$




$$
\leq c \int_{R_{m+k, k}^{+}} \frac{u_{\xi, s}(y) d \mu_{n, k}(y)}{\left(\left|{ }_{s} x^{\prime}-{ }_{s} y^{\prime}\right|+{ }_{s} x \mid\right)^{\beta}} \leq c I_{\beta, s}(u, \xi)(x)<+\infty
$$

Now let us prove estimation $(\Omega)$ of theorem 1 . Take $\xi>0$.

We have

$$
\begin{aligned}
& \Omega_{q, \mu_{n, k}}^{\left({ }_{s} x\right)}(u, \xi) \leq\left\{\int_{\left\{x \in R_{m+k, k}^{+}:|s x| \geq \xi\right\}}\left|A\left(u_{\xi / 2, s}(x)\right)\right|^{q} d \mu_{\gamma_{n, k}}(x)\right\}^{1 / q}+ \\
& +\left\{\int_{\left\{x \in R_{m+k, k}^{+}:\left.\right|_{s} x \mid \geq \xi\right\}}\left|A\left(\bar{u}_{\xi / 2, s}(x)\right)\right|^{q} d \mu_{\gamma_{n, k}}(x)\right\}^{1 / q}=i_{1}+i_{2} .
\end{aligned}
$$

By the condition $A \in \bar{K}_{\nu}(p, q)$ we get

$$
\begin{gathered}
i_{2}=\left\{\int_{\left\{x \in R_{m+k, k}^{+}:\left.\right|_{s} x \mid \geq \xi\right\}}\left|A\left(\bar{u}_{\xi / 2, s}(x)\right)\right|^{q} d \mu_{\gamma_{n, k}}(x)\right\}^{1 / q} \leq \\
\leq c\left\{\int_{\left\{x \in R_{m+k, k}^{+}:\left.\right|_{s} x \mid \geq \xi\right\}}\left|\bar{u}_{\xi / 2, s}(x)\right|^{q} d \mu_{\gamma_{n, k}}(x)\right\}^{1 / q} \leq c \Omega_{p, \mu_{n, k}}^{(s x)}(u, \xi / 2) \leq \\
\leq c \xi^{-\alpha_{p, s}} \int_{0}^{t^{\alpha_{p, s}-1} \Omega_{p, \mu_{n, k}}^{(s x)}(u, t) d t .}
\end{gathered}
$$

For $i_{1}$, using the estimation

$$
\left|A u_{\xi / 2, s}(x)\right| \leq c I_{\beta, s}(u, \xi / 2)(x)
$$

and applying lemma 1 and lemma 2, we get

$$
i_{1} \leq c \xi^{-\alpha_{p, s}} \int_{0}^{\xi} t^{\alpha_{p, s}-1} \Omega_{p, \mu_{n, k}}^{\left(s_{s}\right)}(u, t) d t .
$$

Theorem 1 is proved. 
For further reasonings we need two lemmas.

Lemma 3. Let $\phi \in N$ and $\omega^{p}(t)=\int_{0}^{t} \varphi(\xi) d \xi \quad t>0$. Then

$$
I_{p, \mu_{n, k}}^{(s x)}(\phi)=L_{p, \nu}\left(\omega(|s x|), R_{m+k, k}^{+}\right)
$$

and the appropriate norms are equivalent.

Proof.

$$
\begin{gathered}
\int_{0}^{\infty}\left(\Omega_{p, \mu_{n, k}}^{\left({ }_{s} x\right)}(u, \xi)^{p}\right) \varphi(\xi) d \xi= \\
=\int_{0}^{\infty} \int_{R_{m+k, k}^{+}}\left|u\left({ }_{s} y,{ }_{s} y^{\prime}\right)\right|^{p} \chi_{[0, \xi]}\left(\left|{ }_{s} y\right|\right) d \mu_{\gamma_{n, k}}(y) \varphi(\xi) d \xi= \\
\left.=\int_{\left|u\left({ }_{s} y,{ }_{s} y^{\prime}\right)\right|^{p}\left(\int_{0}^{\infty} \chi_{[0, \xi]}\left(\left|{ }_{s} y\right|\right) \varphi(\xi) d \xi\right.}\right)^{d} d \mu_{\gamma_{n, k}}(y)= \\
=\int_{R_{m+k, k}^{+}}\left|u\left({ }_{s} y,{ }_{s} y^{\prime}\right)\right|^{p}\left(\int_{0}^{\left|{ }_{s} y\right|} \varphi(\xi) d \xi \mid d \mu_{\gamma_{n, k}}(y)=\right. \\
=\int_{R_{m+k, k}^{+}}\left|u\left({ }_{s} y,,_{s} y^{\prime}\right)\right|^{p} \omega^{p}\left(\left|{ }_{s} y\right|\right) d \mu_{\gamma_{n, k}}(y) .
\end{gathered}
$$

Lemma 4. Let $\phi \in N$ and the following integral converge

$$
\int_{0}^{\xi}\left|\varphi^{1 / p}(t) t^{\left(1-\alpha_{p, s}\right)}\right|^{-p^{\prime}} d t<\infty, \quad \alpha_{p, s}=\left(s+\left|\gamma_{k_{s}}\right|\right) / p^{\prime}
$$

Then

$$
I_{p, \mu_{n, k}}^{\left({ }_{s} x\right)}(\varphi) \subset J_{p, \mu_{n, k}}\left({ }_{s} x\right)
$$

Proof.

$$
\int_{0}^{\xi} t^{\alpha_{p, s}-1} \Omega_{p, \mu_{n, k}}^{\left(s_{s} x\right)}(u, t) d t=\int_{0}^{\xi} \Omega_{p, \mu_{n, k}}^{\left({ }_{s} x\right)}(u, t)[\varphi(t)]^{1 / p}[\varphi(t)]^{-1 / p} t^{\alpha_{p, s}-1} d t \leq
$$




$$
\leq \int_{0}^{\xi}\left(\Omega_{p, \mu_{n, k}}^{\left(s_{s} x\right)}(u, t)\right)^{p} \varphi(t) d t=\left(\int_{0}^{\xi}\left([\varphi(t)]^{-1 / p} t^{\alpha_{p, s}-1}\right)^{p^{\prime}} d t\right)^{1 / p^{\prime}}<\infty .
$$

Proof of Theorem 2. By $(\varphi, \psi)$ the integral in the conditions of lemma 4 converges, whence by lemma $5 I_{p, \infty, i}(\varphi) \subset J_{p, j}\left(x_{i}\right)$, and therefore theorem 1 holds.

Assume

$$
\bar{u}(\xi)=\xi^{-\alpha_{p, s}} \psi^{\frac{1}{q}}(\xi) \text { and } \bar{\nu}(\xi)=\varphi^{1 / p}(\xi) \xi^{\left(1-\alpha_{p, s}\right)}
$$

then

$$
\psi(\xi)=\left(\bar{u}(\xi) \xi^{\alpha_{p, s}}\right)^{\chi} \text { and } \varphi(\xi)=\left(\bar{\nu}(\xi) \xi^{\left(\alpha_{p, s}-1\right) p}\right) .
$$

Then by estimation $(\Omega)$, applying the Hardy theorem, we get

$$
\begin{aligned}
& \left(\int_{0}^{\infty}\left(\Omega_{q, \mu_{n, k}}^{(s x)}(u, \xi)\right)^{p} \psi(\xi) d \xi\right)^{\frac{1}{q}} \\
& \leq c\left(\int_{0}^{\infty}\left(\xi^{-\alpha} \int_{0}^{\xi} t^{\alpha-1} \Omega_{p, \mu_{n, k}}^{\left(s_{x} x\right)}(u, t) d t\right)^{q} \psi(\xi) d \xi\right)^{\frac{1}{q}} \\
& =c\left(\int_{0}^{\infty}\left(\xi^{-\alpha} \psi^{\frac{1}{q}}(\xi) \int_{0}^{\xi} t^{\alpha-1} \Omega_{p, \mu_{n, k}}^{\left({ }_{s} x\right)}(u, t) d t\right)^{q}\right)^{\frac{1}{q}} \\
& =c\left(\int_{0}^{\infty}\left(\bar{u}(\xi) \int_{0}^{\xi} t^{\alpha-1} \Omega_{p, \mu_{n, k}}^{\left({ }_{s} x\right)}(u, t) d t\right)^{q}{ }^{\frac{1}{q}}\right)^{\frac{1}{x^{2}}} \\
& \leq c\left(\int_{0}^{\infty} \bar{\nu}^{p}(\xi)\left(\xi^{\alpha-1} \Omega_{p, \mu_{n, k}}^{\left({ }_{s} x\right)}(u, \xi)\right)^{p} d \xi\right)^{\frac{1}{p}}= \\
& =c\left(\int_{0}^{\infty} \bar{\nu}^{p}(\xi) \xi^{(\alpha-1) p}\left(\Omega_{p, \mu_{n, k}}^{\left({ }_{s} x\right)}(u, \xi)\right)^{p} d \xi\right)^{\frac{1}{p}}
\end{aligned}
$$




$$
=\left(\int_{0}^{\infty} \varphi(\xi)\left(\Omega_{p, \mu_{n, k}}^{\left({ }_{s} x\right)}(u, \xi)\right)^{p} d \xi\right)^{\frac{1}{p}}
$$

Theorem 2 is proved.

Let all conditions of theorem 3.1 be fulfilled.

Assume $\varphi(\xi)=\omega(\xi)^{p} \xi^{-1}$ and $\psi(\xi)=\omega_{1}^{q}(\xi) \xi^{-1}$, then the condition

$$
\sup _{t>0}\left(\int_{0}^{\infty}\left|\xi^{-\alpha_{p, s}} \psi^{\frac{1}{q}}(\xi)\right|^{q}\right)^{1 / q}\left(\int_{0}^{\infty}\left|\varphi^{1 / p}(\xi) \xi^{\left(1-\alpha_{p, s}\right)}\right|^{-p^{\prime}}\right)^{1 / p^{\prime}}<\infty
$$

of theorem 2.1 has the form

$$
\sup _{t>0}\left(\int_{t}^{\infty}\left(\xi^{-\alpha_{p, s}} \omega_{1}(\xi)\right)^{q} \frac{d \xi}{\xi}\right)^{1 / q}\left(\int_{0}^{\infty}\left(\omega(\xi) \xi^{-\alpha_{p, s}}\right)^{-p^{\prime}} \frac{d \xi}{\xi}\right)^{1 / p^{\prime}}<\infty
$$

And therefore, theorem 3 is the corollary of theorem 2. Note that theorem 3 may be proved without the condition $\omega$ as well (see the paper [12]).

\section{References}

[1] Abdullayev S.K., Babayev A.A. Some estimations for a singular integral with summable density. DAN SSSR, 188 (2), (1969) 263-265.

[2] Abdullayev S.K., Babayev A.A. On a singular integral with summable densicty, Funksionalniy analiz i ego priminenie. Baku, (1978) 3-32.

[3] Abdullayev S.K., Babayev A.A. On some classes of integral operators in space of summable function, DAN SSSR, 283 (4), (1985) 777-780.

[4] Levitan B.M., Expansion in series by Bessel functions, and Fourier integrals, Uspekhi Mat. Nauk, 6 (2), (1951) 102-143.

[5] Levitan B.M., Generalized shift operators and some applications. M. Cos. Miz. matlit. (1962) $323 \mathrm{p}$.

[6] Kipriyanov I.A., Singular Elliptic Boundary Value Problems, M. Nauka, Fizmatlith, (1997).

[7] Kipriyanov I.A., Klyuchanchev M.I., Estimation of surface potential generated by generalized shift operator. Dokl. AN SSSR, 188 (5), (1969) 97-100.

[8] Kipriyanov I.A., Ivanov L.A., Obtaining fundamental solutions for homogeneous equations with singularities in several variables, Proc. of S.L. Sobolev Workshop, Novosibirsk, 1, (1983) 55-77.

[9] Aliyev I.A., Gadjiev A.D. Weight estimations of singular integrals generated by generalized shift operator, Mathematicheskiy sbornik, 183 (9), (1992) 45-66. 
[10] Abdullayev S.K., Karamaliyev N.R. Weight estimations of singular, weakly singular operators, maximum and fractional maximum functions associated with generalized shift, Proc. Of the IV International Symposium "Fourier series and their Applications". Rostovna-Donu 28 may-3 june, (2006) 44-52.

[11] Abdullayev S.K., Akbarov A.A., Kerimov M.K., Some estimates of singular operators and weakly singular integrals generated by generalized shift. Proceedings of International Conference on a theme "The actual problems of mathematics and computer science" Baku, 14-15.01.2009, 143-150.

[12] Abdullayev S.K., Akperov A.A., Kerimov M.K. Two-weight estimates for sublinear operators associated with Laplace-Bessel differential operator. Vestnik Bryanskogo Gosudarstvennogo Univ. ser. exact and natural sciences. (4), (2009) 6-14.

[13] Abdullayev S.K., Gadjieva E.A., Isayev F.A. Two-weighted inequality for $(p, q)$ admissible $B_{k, n}$-potential operators in weighted Lebesgue spaces. Fractional Differential Calculus, (2), (2016) 233-248.

[14] Sobolev S.L. On one functional analysis theorem, Math. Sb., Russia, (1938). 
